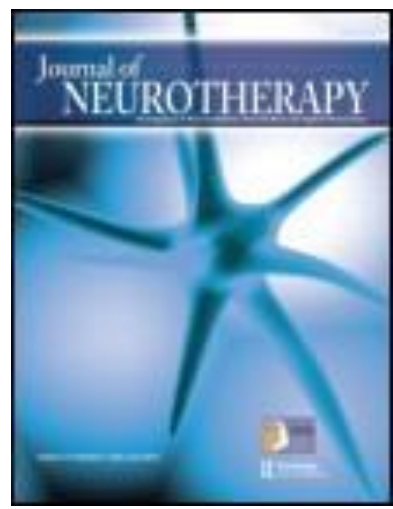

Journal of Neurotherapy: Investigations in Neuromodulation, Neurofeedback and Applied Neuroscience

\title{
Limbic Beta Activation and LORETA: Can Hippocampal and Related Limbic Activity Be Recorded and Changes Visualized Using LORETA in an Affective Memory Condition?
}

\author{
Rex Cannon BA ${ }^{a}$, Joel Lubar PhD ${ }^{b}$, Keri Thornton BA ${ }^{b}$, Stuart Wilson BA ${ }^{b}$ \& Marco \\ Congedo $\mathrm{PhD}^{\mathrm{C}}$ \\ ${ }^{a}$ experimental psychology program , University of Tennessee, Brain Research and \\ Neuropsychology Lab , Knoxville, TN \\ ${ }^{\mathrm{b}}$ University of Tennessee, Knoxville \\ ${ }^{\mathrm{C}}$ National Institute for Research in Informatics and Random Systems (IRISA), France \\ Published online: 08 Sep 2008.
}

To cite this article: Rex Cannon BA, Joel Lubar PhD , Keri Thornton BA, Stuart Wilson BA \& Marco Congedo PhD (2004) Limbic Beta Activation and LORETA: Can Hippocampal and Related Limbic Activity Be Recorded and Changes Visualized Using LORETA in an Affective Memory Condition?, Journal of Neurotherapy: Investigations in Neuromodulation, Neurofeedback and Applied Neuroscience, 8:4, 5-24

To link to this article: http://dx.doi.org/10.1300/J184v08n04_02

\section{PLEASE SCROLL DOWN FOR ARTICLE}

(C) International Society for Neurofeedback and Research (ISNR), all rights reserved. This article (the "Article") may be accessed online from ISNR at no charge. The Article may be viewed online, stored in electronic or physical form, or archived for research, teaching, and private study purposes. The Article may be archived in public libraries or university libraries at the direction of said public library or university library. Any other reproduction of the Article for redistribution, sale, resale, loan, sublicensing, systematic supply, or other distribution, including both physical and electronic reproduction for such purposes, is expressly forbidden. Preparing or reproducing derivative works of this article is expressly forbidden. ISNR makes no representation or warranty as to the accuracy or completeness of any content in the Article. From 1995 to 2013 the Journal of Neurotherapy was the official publication of ISNR (www. Isnr.org); on April 27, 2016 ISNR acquired the journal from Taylor \& Francis Group, LLC. In 2014, ISNR established its official open-access journal NeuroRegulation (ISSN: 2373-0587; www.neuroregulation.org).

THIS OPEN-ACCESS CONTENT MADE POSSIBLE BY THESE GENEROUS SPONSORS
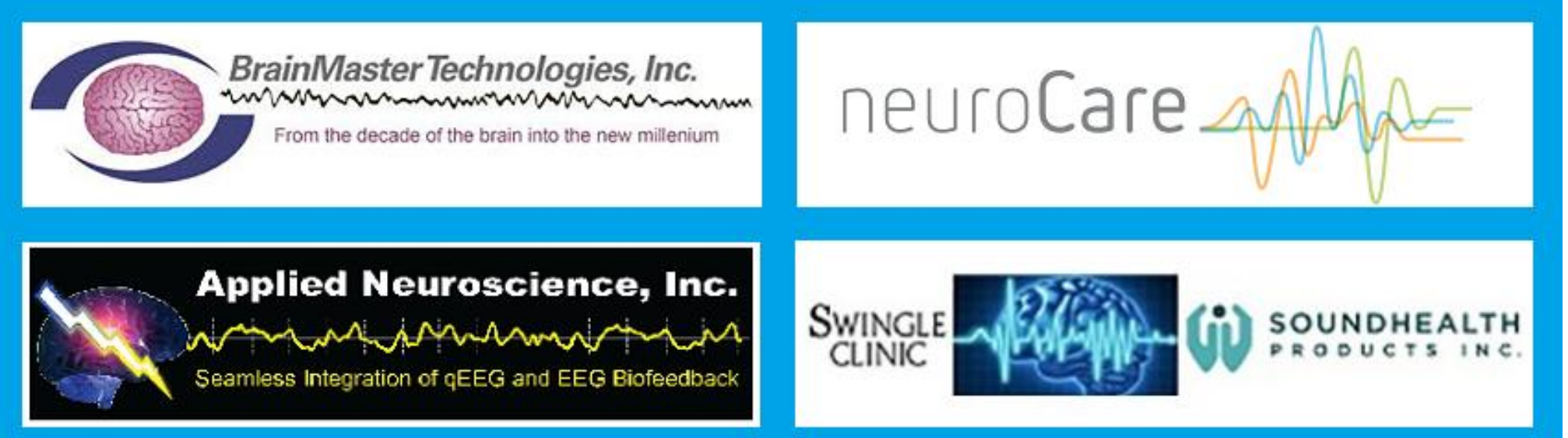
SCIENTIFIC ARTICLES

\title{
Limbic Beta Activation and LORETA: Can Hippocampal and Related Limbic Activity Be Recorded and Changes Visualized Using LORETA in an Affective Memory Condition?
}

\author{
Rex Cannon, BA \\ Joel Lubar, PhD \\ Keri Thornton, BA \\ Stuart Wilson, BA \\ Marco Congedo, $\mathrm{PhD}$
}

Rex Cannon is a graduate student working toward a doctoral degree in the experimental psychology program at the University of Tennessee, Brain Research and Neuropsychology Lab, Knoxville, TN. Joel Lubar is Professor at the University of Tennessee, Knoxville. Stuart Wilson, and Keri Thornton are students at the University of Tennessee, Knoxville. Marco Congedo is a post-doctoral fellow at the National Institute for Research in Informatics and Random Systems (IRISA) in France.

Address correspondence to: Rex Cannon, University of Tennessee, Department of Psychology, Brain Research and Neuropsychology Laboratory, Knoxville, TN 37996 (E-mail: rcannon2@utk.edu).

The authors give special thanks to Dr. John C. Malone, Dr. Deborah Baldwin, and Jesse Rothove, BA, for sharing their knowledge, time, and editorial assistance.

Journal of Neurotherapy, Vol. 8(4) 2004

Copyright (C) 2004 ISNR. All rights reserved.

Digital Object Identifier: 10.1300/J184v08n04_02 
ABSTRACT. Background. The purpose of this study was to determine the validity of Low Resolution Electromagnetic Tomography (LORETA) in visualizing limbic structures and possibly identifying electroencephalographic (EEG) frequencies in the limbic region during an anger memory recall process.

Method. This study was conducted with twelve subjects, non-clinical students at the University of Tennessee, Knoxville. A pre-study screening was conducted. Eyes-open baselines were obtained employing 300 epochs, or five minutes, using a 19-channel quantitative electroencephalographic (qEEG) acquisition system with linked ear reference. The experimental condition recording directly followed an eyes-open baseline. The experimental condition was to allocate a memory that created intense anger and retain the state as long as possible. All files were no less than 100 total epochs upon editing. The data were analyzed in both individual and group conditions with LORETA imaging software. Statistical differences between conditions were evaluated for significance, then computed and transformed into LORETA images.

Results. The data revealed significant differences between the anger condition and baseline recordings in limbic structures and frontal regions. The data suggests that limbic lobe and hippocampal activity can be recorded and visualized using LORETA during affective memory recall. There are several notable differences between the baseline and condition images. One of the more interesting of these differences is possible activation of the amygdala, uncinate gyrus and surrounding structures in the beta $(12-32 \mathrm{~Hz})$ frequencies. The hemispheric asymmetries during anger memory recall offer further support for the lateralization of hemispheric activity relating to affective states.

Conclusion. LORETA may be an effective method used to differentiate and visualize limbic lobe, hippocampal formation and other related structures during affective anger memory recall.

KEYWORDS. LORETA, amygdaloid complex, limbic lobe, hippocampus, anger, emotional lateralization, mnemonic processes, qEEG, affective cortical processes, mid-brain beta activation

\section{INTRODUCTION}

Research pertaining to the amygdala and hippocampal region is extensive; however, no studies have been conducted on this sub-cortical 
area using Low Resolution Electromagnetic Tomography (LORETA) during affective (anger) memory recall. Moreover, studies specific to the amygdala and hippocampus often use external stimuli or a combination of emotional processes rather than focusing on one specific selfgenerated emotion. The amygdala and hippocampal formation are reported to be involved in declarative memory and emotional processes; however, to date activation of either structure in electroencephalographic (EEG) frequencies remains elusive. The purpose of this study was to inquire as to the validity of LORETA in visualizing limbic structures and possibly identifying EEG frequencies in the limbic region during an affective anger memory process.

LORETA (Pascual-Marqui, 1995, 1999, 2000; Pascual-Marqui, Michel, \& Lehmann, 1994; Pascual-Marqui, Esslen, Kochi, \& Lehmann, $2002 \mathrm{a}, 2002 \mathrm{~b}$ ) is currently the most accepted inverse solution procedure (Congedo, 2003), and has been widely used in electrophysiological studies (Bosch-Bayard et al., 2001, Isotani et al., 2001; Pizzagalli et al., 2002; Lehmann et al., 2001; Pascual-Marqui et al., 1999) and has been used in numerous laboratories (Fernández-Bouzas et al., 1999; Fuchs, Wagner, Köhler, \& Wischmann, 1999; Gomez \& Thatcher, 2001; Prichep, John, \& Tom, 2001; Worrell et al., 2000). The inverse problem of neurophysiology suggests that electroencephalogram/magnetoencephalogram (EEG/MEG) measurements do not provide enough information for ascertaining the neuronal activity distribution in the volume the brain occupies (Pascual-Marqui et al., 1994). LORETA approximates the distribution of electrical neuronal activity in three-dimensional space, basing the approximations on a dense grid of electrodes positioned over the entire scalp (Congedo, 2003). The EEG is a measure of electric potential variations. LORETA estimates the current density that results in a quantifiable potential divergence or the source of the electric field on the scalp (EEG), for which it is feasible to co-register the resolution to a brain atlas (Lancaster et al., 1997, 2000; Talairach \& Tournoux, 1988; Towle at al., 1993), and map electrical activity in all cortical structures (Congedo, 2003). LORETA current density is mapped in a space of $2394,7 \times 7 \times 7 \mathrm{~mm}^{3}$ voxels.

Many of the most recent studies use event-related functional magnetic resonance imaging (fMRI) techniques and often utilize external stimuli to elicit emotive cortical activity. Damasio et al. (2000) performed a positron emission tomography (PET) study on subcortical areas during self-generated emotions, including anger. The results suggest that during self-generated emotional events, limbic and cortical structures that regulate or influence homeostasis are activated. Their study 
reported activation of the insular and cingulate cortices and limbic areas during these affective mnemonic events, and suggested a region of emotion-specific neural patterns that receive or project input to thalamic and other sub-cortical structures. Maratos, Dolan, Morris, Henson, and Rugg (2001) suggest that cortical regions activated by environmental factors are also activated when affective memories are accessed. LORETA methodology has chiefly been exploited to visualize activation in the cortex. Isotani et al. (2001) reported differences between negative, neutral and joyful states in the left-posterior regions of the cortex and parahippocampal gyrus using LORETA, and also noted a decrease in theta activity during a joyful state as compared with a sad state. Studies involving the hippocampal formation and amygdala suggest that both of these structures and the neocortex have different patterns of activation that are dependent on the subject's state of vigilance (Pare, Collins, \& Pelletier, 2002).

The amygdala is located in the anterior medial portion of each temporal lobe and is suggested to be involved with declarative memory and emotions associated with mnemonic events (Kolb \& Whishaw, 1996). It is suggested to have extensive, complex connections to the prefrontal and frontal cortex and thalamo-cortical structures, and is suggested to be part of a circuit mediating fear responses (Rosenzweig, Leiman, \& Breedlove, 1996). The parahippocampal gyrus forms a large part of the limbic lobe along the ventromedial part of the temporal cortical mantle. It has entorhinal and perirhinal sub-divisions and is suggested to be directly involved in both declarative and non-declarative memory processes (Kolb \& Whishaw, 1996). The uncinate gyrus is medial to the amygdala. The amygdala causes a bulge (uncus) in the medial part of the temporal lobe. There is little information on these two structures in current literature. Research suggests that the amygdala and frontal cortex showed an increased coherence of theta activity associated with arousal, and that arousal is accompanied by increases in amplitude of 'fast focal activities and amygdala spindles' and this increase in 'beta/ gamma activity' is associated with different behavioral states (Pare et. al., 2002). Damasio et al. (2000) suggest that emotions of happiness, sadness, fear and anger activate or deactivate structures that influence homeostasis, such as the insular cortex, secondary somatosensory cortex, and the anterior and posterior regions of the cingulate gyrus. Their experiment did not report activation of the amygdala for any of the conditions in the experiment. In experiments with monkeys, research suggests that the hippocampus is involved with spatial and other forms of declarative memory and the amygdala is involved in memory where 
there is an emotional context (Kolb \& Whishaw, 1996). EEG studies of emotion offer support that hemispheric activity differs during affective states (Rosenzweig et al., 1996; Ahern \& Schwartz, 1979), with left activation during more positive emotions and the right for more negative affective processes.

\section{METHODS}

Participants. The participants consisted of twelve subjects, eight female and four male non-clinical students at the University of Tennessee, Knoxville with a mean age of 23. Ten of the subjects were righthanded and two were left-handed. Subjects were excluded under the conditions of previous head trauma, history of epilepsy, drug or alcohol use and any previous psychiatric diagnosis. The subjects received extra credit points for participating in this study. All participants read and signed an informed consent form.

Procedure. Participants were prepared for EEG recording using a measure of the distance between the nasion and inion to determine the appropriate cap size for recording (Electrocap, Inc; Blom \& Anneveldt, 1982.). The ears and forehead were cleaned for recording with Nuprep, a mild abrasive gel used to remove any oil and dirt from the skin. The caps were fitted and each electrode site was injected with electrogel and prepared so that impedances between individual electrodes and each ear were between 3 and $5 \mathrm{~K} \Omega$. The EEG data were then collected using the standard international 10/20 system (FP1, FP2, F3, F4, Fz, F7, F8, C3, C4, Cz, T3, T4, T5, T6, P3, P4, Pz, O1 and O2) and the Lexicor Medical Technology, Inc. Neurosearch-24 EEG analog to digital acquisition system. The data was collected and stored using a Pentium III computer with Lexicor's V-41e software with a band pass set at $0.5-64.0 \mathrm{~Hz}$ for 256 samples per second. All recordings obtained in this study were in the eyes-opened resting condition and were conducted in a comfortably lit, sound attenuated room in the Neuropsychology and Brain Research Laboratory at the University of Tennessee, Knoxville. Both the eyesopen baselines and experimental condition recordings were obtained for 300 epochs or five minutes. Subjects were instructed to control eye movements, blinks and muscle activity from forehead, neck and jaws. The experimental condition recordings were obtained immediately following baselines. The subjects were asked to recall and retain a memory that created intense anger and hold the experience as long as possible. 
All subjects reported being able to hold the experience for the length of the recording. The subjects were oblivious to the experimental condition under the hypothesis that a spontaneous memory would be more valid than a planned or suggested memory. The recordings were taken using typical frequencies: delta $=0.5-3.5 ;$ theta $=4.0-7.5 ;$ Lalpha $=$ 8.0-10.0; Halpha = 10.0-12.0; Lbeta $=12.0-21.0$; beta $=21.0-32.0$; gamma $=32.0-42.0$, respectively. The frequencies within beta band were calculated: beta $1(12-16 \mathrm{~Hz})$; beta $2(16-20 \mathrm{~Hz})$; beta $3(20-24$ $\mathrm{Hz})$; beta $4(24-28 \mathrm{~Hz})$; beta $5(28-32 \mathrm{~Hz})$. These beta frequencies were extracted in order to observe more specific ranges of activity.

Data Analysis. The data collected was stringently artifact rejected; eye blinks, eye movements, teeth clenching, jaw tension, body movements and possible EKG were removed from the EEG stream using Eureka3! software (Nova Tech EEG, Inc.). All files were no less than 100 total epochs after editing. The EEG stream was edited as suggested by Thornton (1996). LORETA current density was extracted in all bands using the LORETA-Key package (Key Institute for Brain-Mind Research). Current density for total relative and absolute power was also extracted. The statistical data was analyzed using the Multiple Hypothesis Testing (MhyT3!) software (Nova Tech EEG, Inc.). The procedure used is known as the t-max test using the step-down version of the procedure with 1000 and 5000 random data permutations (Blair \& Karniski, 1994; Holmes, Blair, Watson, \& Ford, 1996, Westfall \& Young, 1993). The statistical differences within and between conditions were corrected for all multiple comparisons using non-parametric analysis. Baseline and experimental condition files were contrasted by means of voxel by voxel t-statistics. All data was normalized, log transformed and smoothed with a $21 \mathrm{~mm}$ moving average filter (MAF 3-D) before entering statistical analysis. Type one error $(<0.05)$ was controlled for using family wise error in the strong sense by means of permutation theory and maximum t-statistics (Pesarin, 2001). The specific contrast between conditions was entered into one sample t-test corresponding to a corrected $\mathrm{P}<.05$. All voxels were examined and statistical data was transformed into LORETA images.

\section{RESULTS}

Statistical Data. The average cross-spectral matrices were calculated for each subject in this study and statistical representations were transformed into LORETA images for the classical bands mentioned earlier. 
The cross-spectral matrices (FFT) were computed and averaged over four-second epochs, which resulted in one cross-spectral matrix for each subject and for each discrete frequency within each band. LORETA current density is computed directly from the cross-spectral matrix and incorporates a three-shell spherical head model registered to the Talairach and Tournoux (1988) anatomical brain atlas and makes use of EEG electrode coordinates derived from the cross-registration between spherical and realistic head geometry (Towle et al., 1993). LORETA has a very limited range of voxels for localization of activity in the limbic region; especially regarding the hippocampus and amygdala. Therefore, identification of artifact-free activity in these regions suggests that it is authentic. Statistical data were analyzed by two separate methods: primarily with the multiple hypotheses testing software (Nova Tech EEG, Inc.) and secondly, with non-parametric testing software (LORETA, Key Institute for Brain-Mind Research). The results of both paradigms were similar. The maximum cumulative activation reported by the test-statistics is at $(X=25, Y=-4, Z=-20)$, the amygdala, uncus and limbic lobe. Computations were based on the Talairach and Tournoux probability atlases of the Brain Imaging Centre, Montreal Neurologic Institute (2394 voxels; resolution: $7 \mathrm{~mm}^{3}$ ).

Specific Beta Frequencies. The anger condition and baseline recordings differ in many respects. Activity in the theta $(4-8 \mathrm{~Hz})$ and alpha $(8-12 \mathrm{~Hz})$ frequencies appear in all recordings in similar regions, however, they appear to vary in intensity. The differences in alpha $(8-12 \mathrm{~Hz})$ and beta $(12-32 \mathrm{~Hz})$ specifically appear in the inferior-right-frontal sections, hippocampus, parahippocampal gyrus, amygdala, uncus and insular cortex.

Figure 1 is a combined statistically significant representation of the differences between the current density of anger condition and baseline recordings. The regions significant in activation are the uncus, amygdala and hippocampal formation (the areas of interest in this study) and frontal areas. There are other areas that deserve consideration such as the periamygdaloid cortex. However, in studies this region is suggested to be more involved with olfactory processes projecting to the ventrolateral and medial divisions of the amygdala, but not the dorsolateral division of the lateral amygdaloid nucleus (Savander, LeDoux, \& Pitkänen, 1996).

Figure 2 shows the significant differences between anger condition and baseline recordings in the beta 1 frequency $(12-16 \mathrm{~Hz})$. This image suggests that the frontal activity is decreased in both hemispheres but more significantly in the left frontal while there is a significant increase 


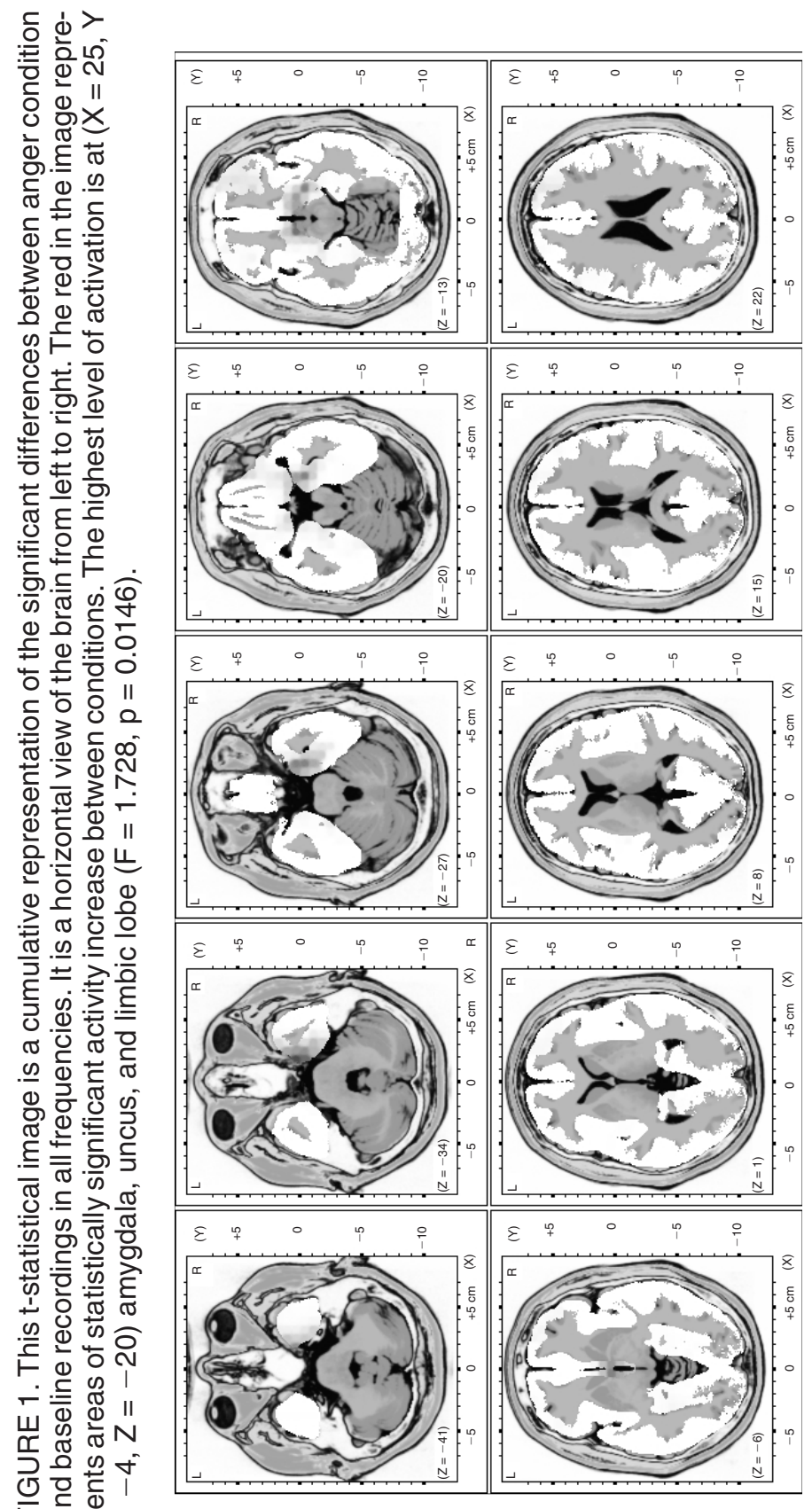




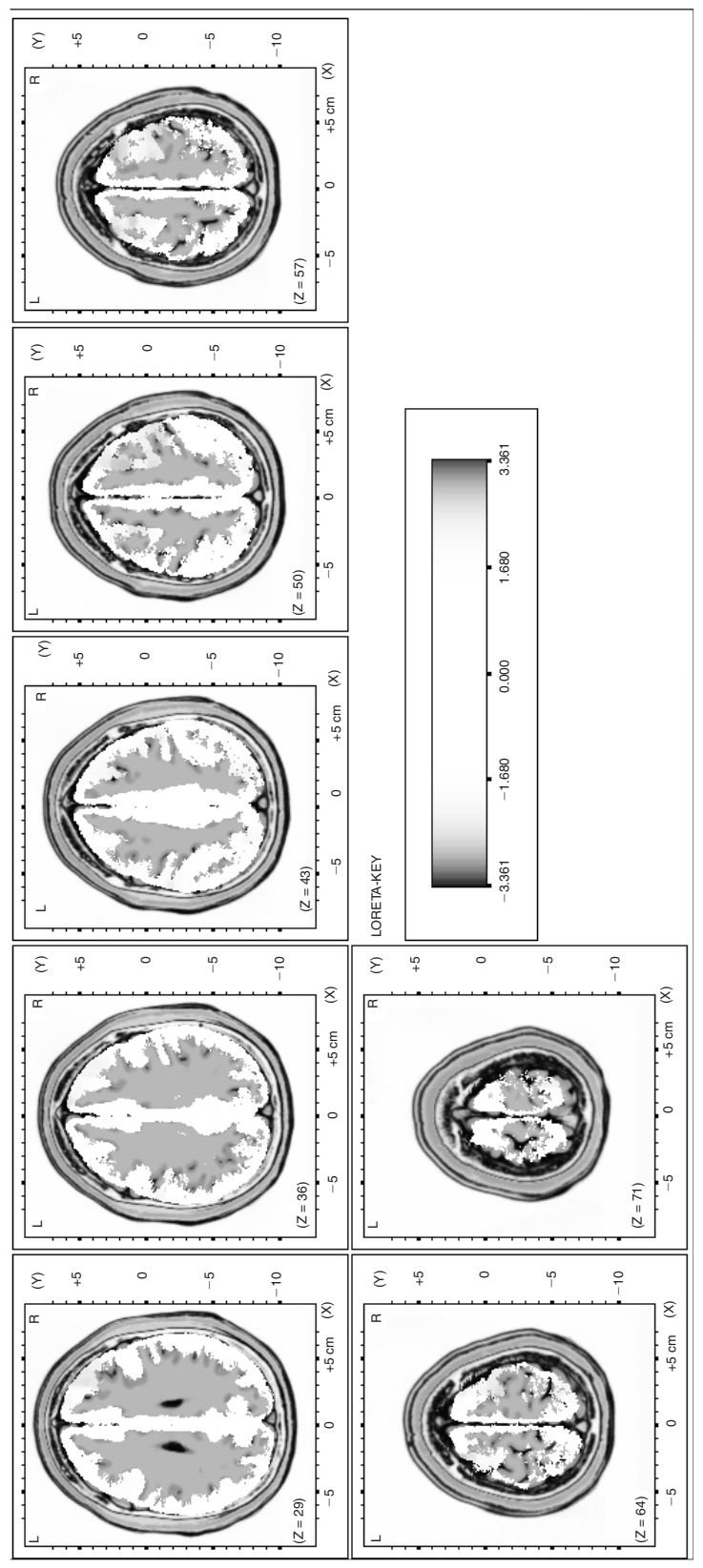




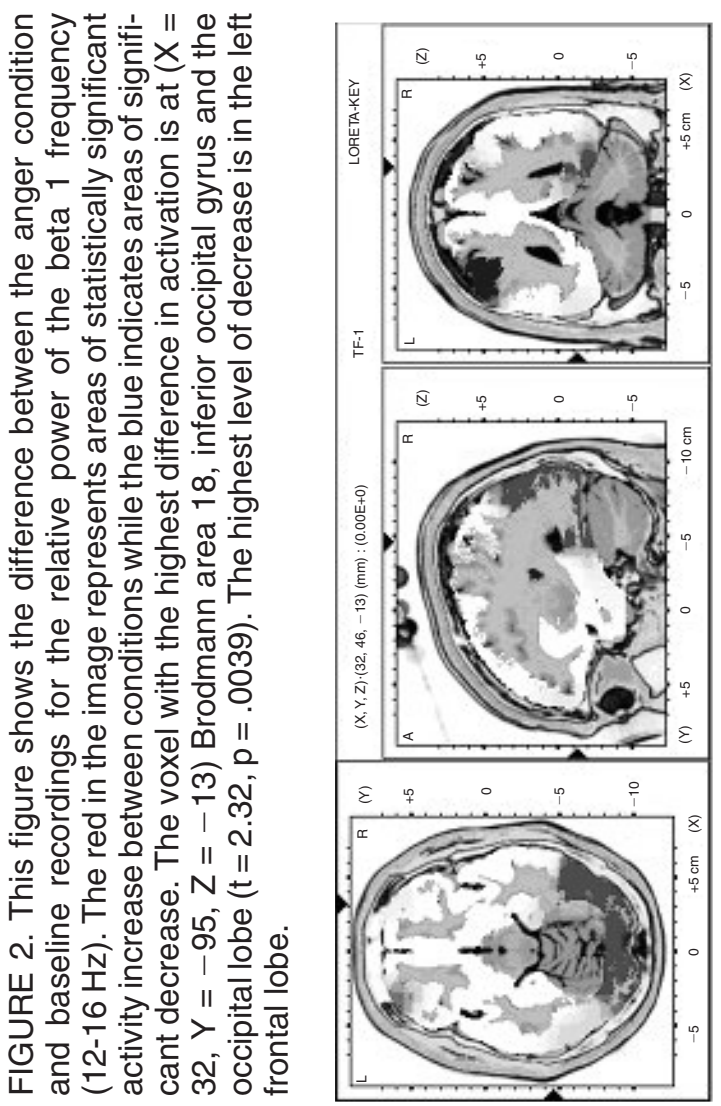


in the right occipital and parietal regions. The significant increase in beta 1 activity can possibly be attributed to the allocation and visualization of the mnemonic event.

Figure 3 shows the statistical differences between the anger condition and baseline recordings in the beta 2 frequency $(16-20 \mathrm{~Hz})$. The beta activity appears more pronounced in the amygdala and hippocampal regions in this frequency, specifically to the amygdala, insular cortex, parahippocampal gyrus, hippocampus, sub-gyral and uncus. It is possible that these structures are primarily involved in an anger/memory circuit. The activity becomes focused in the right frontal lobe in this frequency, which could be attributed to the cognitive evaluation of the stimuli.

Figure 4 shows the differences between anger condition and baseline recordings in the beta 3 frequency (20-24 Hz). The significant differences in this image appear in the uncus, hippocampus, amygdala and parahippocampal gyrus. This activation is possibly attributed to the allocation and processing of the memory and the associated emotion. The decrease in activity in the temporal lobes is not at levels (in scaling) for the blue to appear in the image.

Figure 5 shows the significant differences between the anger condition and baseline recordings in the beta 4 frequency $(24-28 \mathrm{~Hz})$. The significant activation occurs in the inferior frontal gyrus and frontal lobe. Again, this could possibly be a cognitive evaluation of the stimuli creating the emotion. The decrease in activation was not at levels (in scaling) for the blue to register in the image.

Figure 6 shows the difference between the anger condition and baseline recordings in the beta 5 frequency $(28-32 \mathrm{~Hz})$. The activation in this image occurs in the right frontal, anterior and posterior cingulate gyrus and insular cortex. It is possible that the activation in the cingulate gyrus (both anterior and posterior) relates to the suggestion that it is a possible generator or gating mechanism between the limbic system and cortex (Pizzagalli, Oakes, \& Davidson, 2003). The beta frequencies presented the most significant statistical differences between conditions. The regions with the highest statistically significant activation are the right hippocampus, amygdala, uncus, parahippocampal gyrus, insular cortex, sub-gyral, right dorsolateral prefrontal, and frontal lobes. This identification of the amygdaloid complex contrasts studies in which this area was reportedly decreased, non-active or undetectable in a self-generated anger condition (Damasio et al., 2000). Meanwhile the regions most significantly decreased in activity were the left prefrontal, temporal and frontal regions. This lateralization of activation was not a pre- 


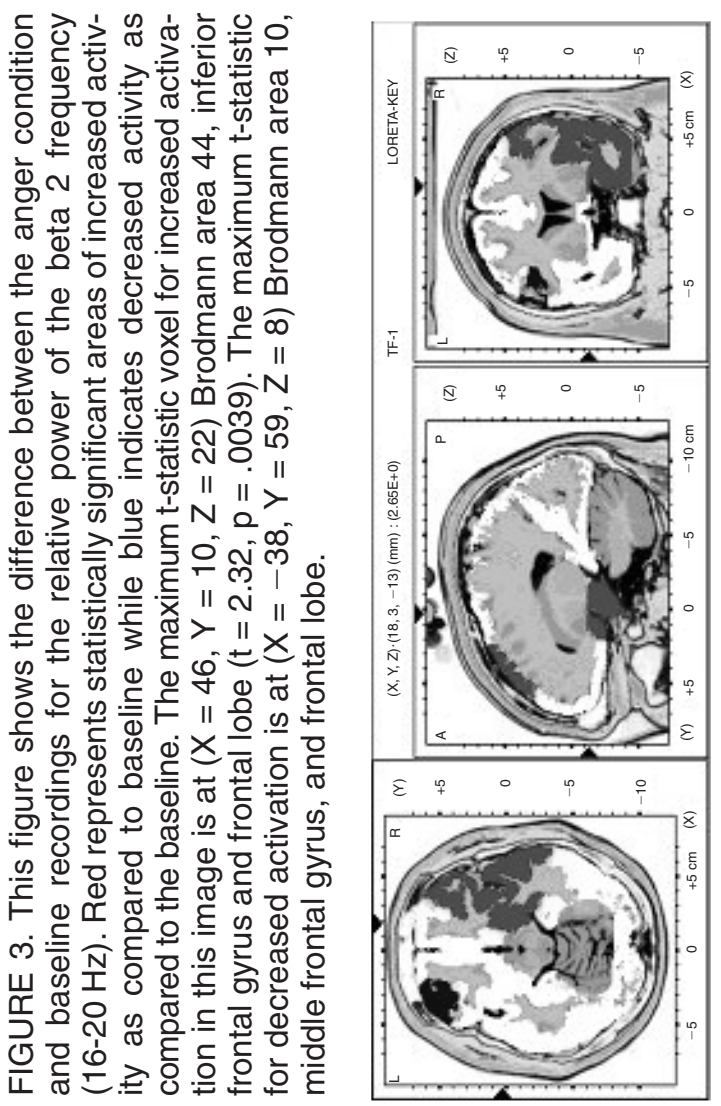




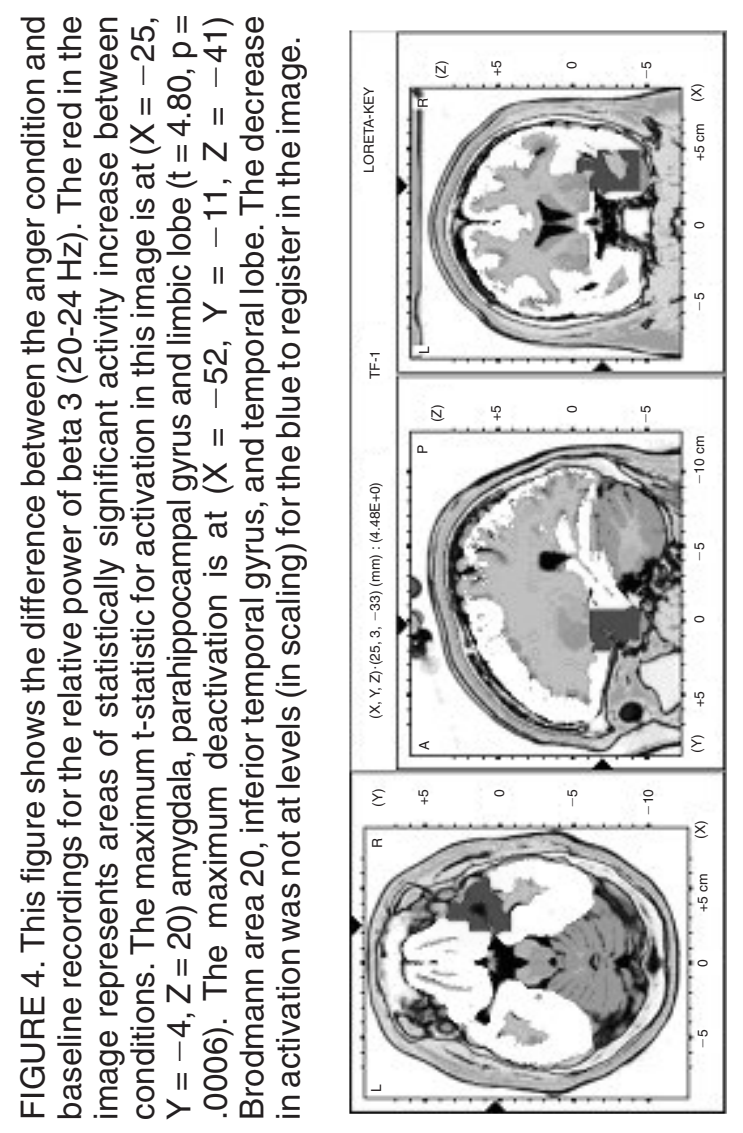




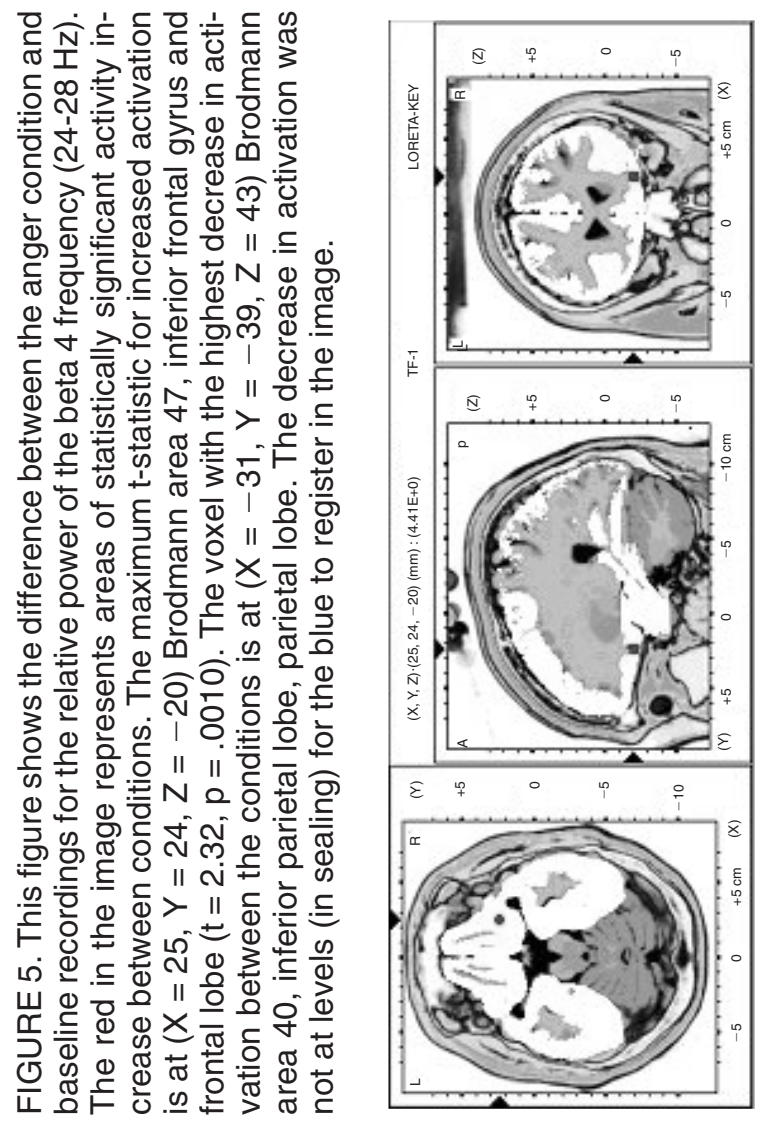




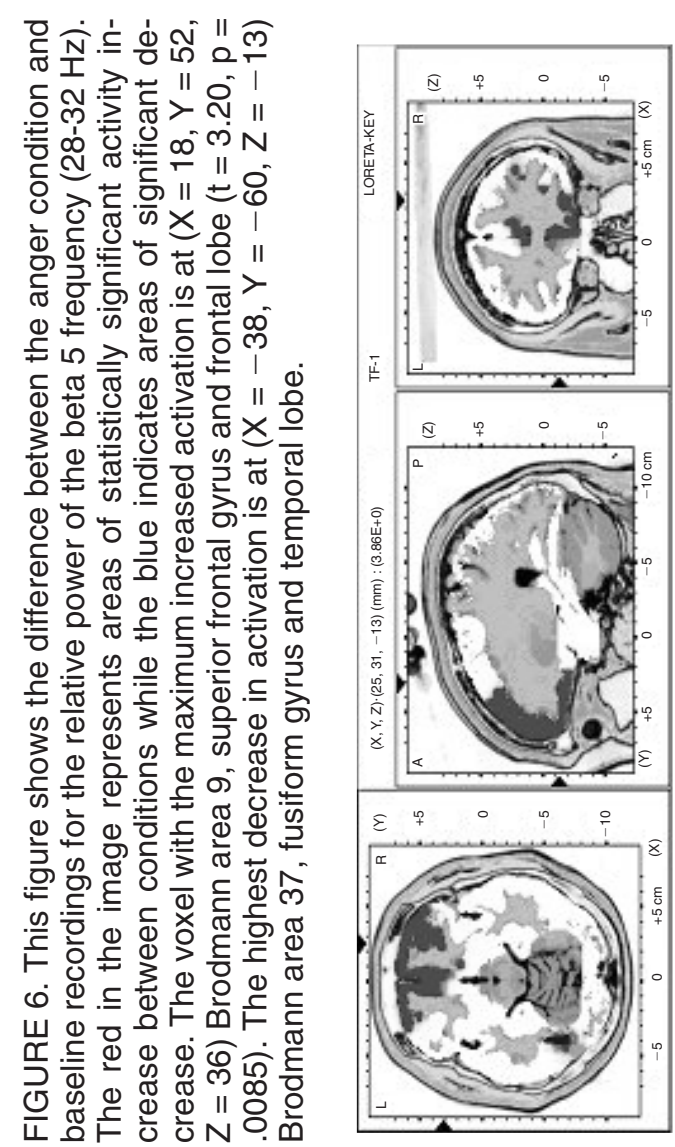


dicted outcome of this study; nonetheless it was observed and contrasts to the meta analysis by Wager, Phan, Liberzon, and Taylor (2003). The reasons for this contrast are unknown at this time; however, they may be attributed to the focus of the experiment being anger from an internal mechanism rather than an external stimuli, or recording one specific emotion rather than a combination of emotions. One benefit to this study could have been to have the participants provide a subjective written record of the memory and to rate the intensity of the event.

\section{DISCUSSION}

The anger condition and baseline recordings show significant differences in many regions. The cortical areas of interest for this study were identified by the data and differences in these regions were statistically significant. Alpha (8-12 Hz) and theta (3.5-7.5 Hz) activity were present in both conditions. However, the intensity of activity differed dependent on the condition-in all cases increased activity occurred during the anger condition recordings. The data suggests that when the subject is in an angered state, the right hemisphere and the limbic structures are more active in the beta frequency while limbic structures in the left hemisphere are less active as are the left frontal areas. The data collected and analyzed suggest that limbic lobe activity including the amygdaloid complex; hippocampus, parahippocampal gyrus, uncus, posterior and anterior regions of the cingulate gyrus can be visualized using LORETA. The highest statistical difference for all frequencies between the anger condition and baselines was given at Talairach coordinates $(X=25, Y=-4, Z=-20)$ amygdala, uncinate gyrus, and limbic lobe. The highest decrease in activity was at $(X=-45, Y=-67$, $\mathrm{Z}=-6$ ) Brodmann area 37 and inferior temporal gyrus. Theta rhythm is suggested to serve as a gating function for information flow in the limbic region and facilitates communication between these structures and the cortex (Pizzagalli, Oakes, \& Davidson, 2003; Pizzagalli, Hendrick, Horras, \& Davidson, 2002). This idea is supported in that the activity in both the anterior and posterior regions of the cingulate gyrus in the range of 4 to $8 \mathrm{~Hz}$ is significant between conditions, with increased activation occurring in the anger condition. Other areas of significant difference are Brodmann areas 21, 13, 29 and the insular cortex. It is interesting that the increase in beta activity occurs principally in the right hemisphere in all the structures in the area of interest in this study. The right amygdaloid complex, hippocampus, insular cortex, parahip- 
pocampal gyrus, uncinate gyrus and sub-gyral appear in most beta frequencies, suggesting that anger may be a predominantly right hemispheric process including the allocation and possible cognitive evaluation of the memory and the associated emotion.

\section{CONCLUSION}

The limbic lobe (or limbic system) has been the subject of numerous studies, and remains a focal point for understanding the complexities of human behavior. Technology has allowed us to visualize activity in this region with functional magnetic resonance imaging (fMRI), positron emission tomography (PET), and computerized axial tomography (CT) methodologies, yet these studies have used mostly task specific orientation for experiments, which fail to maintain activity in the structures for an adequate amount of time. This study was able to record mnemonic activity, anger processes, and the cortical asymmetries between these states and an eyes-open baseline. It also provided an opportunity to evaluate possible generators for emotional cortical events. Both mnemonic and anger events appear to generate alpha $(8-12 \mathrm{~Hz})$, theta (4-8 $\mathrm{Hz})$ but more predominantly beta $(12-32 \mathrm{~Hz})$ activity in the limbic lobe including the amygdaloid complex and hippocampal regions, frontal, pre-frontal and parietal areas discussed earlier. Research suggests that these specific structures are involved in circuits of fear or systems of memory (Kolb \& Whishaw, 1996). It is not implausible to implicate these structures in a possible anger circuit. Lateralization of activity during affective (anger) memory processes was an observed outcome, but not an expected one and contrasts with results by Wager et al. (2003). The reason for this contrast is not known, but one possibility is that this study focused on one emotional element whereas other similar studies use three or more. Damasio et al. (2000) suggest that the reason for lack of amygdaloid complex activity in their study could be attributed to the skewing of data collection by the emotion-feeling cycle rather than induction of emotion; another is the possible habituation of the amygdala during the feeling phase of the experiment. Kolb and Whishaw (1996) suggest that the hippocampus is responsible for spatial and other declarative memories, while the amygdala stores, or is a control station for, the retrieval of the associated emotional content. The combined data suggest that activity in the limbic lobe, hippocampal for- 
mation and other sub-cortical structures may be recorded and changes can be differentiated and visualized in affective (anger) memory processes by using LORETA. The possibilities this study presents are exciting, but further study is important and necessary to confirm these results.

\section{REFERENCES}

Ahern, G. L., \& Schwartz, G. E. (1979). Differential lateralization for positive versus negative emotion. Neuropsychologia, 17, 693-698.

Blair, R. C., \& Karniski, W. (1994) Distribution-free statistical analyses of surface and volumetric maps. In R.W. Thatcher, M. Hallett, E. R. John, \& M. Huerta (Eds.), Functional neuroimaging: Technical foundations, (pp. 114-118). San Diego, CA: Academic Press.

Blom, J. L., \& Anneveldt, M., (1982). An electrode cap tested. Electroencephalography and Clinical Neurophysiology, 54, 591-594.

Bosch-Bayard, J., Valdés-Sosa, P., Virues-Alba, T., Aubert-Vázquez, E., John, E. R., Harmony, T., et al. (2001). 3D statistical parametric mapping of EEG source spectra by means of variable resolution electromagnetic tomography (VARETA). Clinical Electroencephalography, 32, 47-61.

Congedo, M. (2003). Tomographic neurofeedback: A new technique for the self-regulation of brain electrical activity. An unpublished dissertation. University of Tennessee, Knoxville.

Damasio, R. A., Grabowski, T. J., Bechara, A., Damasio, H., Ponto, L. B., Parvizi, J., et al. (2000). Subcortical and cortical brain activity during the feeling of self-generated emotions. Nature Neuroscience, 3 (10), 1049-1050.

Fernández-Bouzas, A., Harmony, T., Bosch-Bayard, J., Aubert-Vázquez, E., Fernández, T., Valdés-Sosa, Silva J., et al. (1999). Source of abnormal EEG activity in the presence of brain lesions. Clinical Electroencephalography, 30, 46-52.

Fuchs, M., Wagner, M., Köhler, T., \& Wischmann, H. (1999). Linear and nonlinear current density reconstructions. Journal of Clinical Neurophysiology, 16 (3), 267295.

Gomez, J. F., \& Thatcher, R. W. (2001). Frequency domain equivalence between potentials and currents using LORETA. International Journal of Neuroscience, 167, 161-171.

Holmes, A. P., Blair, R. C., Watson, J. D. G., \& Ford, I. (1996). Nonparametric analysis of statistic images from functional mapping experiments. Journal of Cerebral Blood Flow and Metabolism, 16, 7-22.

Isotani, T., Lehmann, D., Pascual-Marqui, R. D., Fukushima, M., Saito, N., Yagyu, T., et al. (2001). Source localization of brain electric activity during positive, neutral and negative emotional states. International Congress Series, 1232, 165-173.

Kolb, B., \& Whishaw, I. Q. (1996). Fundamentals of human neuropsychology (4th ed.; pp. 59-168, 289-290, 370-371, 424). New York: W.H. Freeman and Co.

Lancaster, J. L., Rainey, L. H., Summerlin, J. L., Freitas., C. S., Fox., P. T., Evans, A. C., et al. (1997). Automated labeling of the human brain: A preliminary report on the development and evaluation of a forward-transform method. Human Brain Mapping, 5, 238-242. 
Lancaster, J. L., Woldorff, M. G., Parsons, L. M., Liotti, M., Freitas, C. S., Rainey, L., et al. (2000). Automated Talairach atlas labels for functional brain mapping. $\mathrm{Hu}$ man Brain Mapping, 10, 120-131.

Lehmann, D., Faber, P. L., Achermann, P., Jeanmonod, D., Gianotti, L. R. R., \& Pizzagalli, D., (2001). Brain sources of EEG gamma frequency during volitional meditation-induced, altered states of consciousness, and experience of the self. Psychiatric Research: Neuroimaging Section, 108, 111-121.

Maratos, E. J., Dolan, R. J., Morris, J. S., Henson, R. N. A., \& Rugg, M. D. (2001). Neural activity associated with episodic memory for emotional context. Neuropsychologia, 39, 910-920.

Pare, D., Collins, D. R., \& Pelletier, J. G. (2002). Amygdala oscillations and the consolidation of emotional memories. Trends in Cognitive Sciences, 6-7, 306-314.

Pascual-Marqui, R. D. (1995). Reply to comments by Hämäläinen, Ilmonieni, and Nunez. In W. Skrandies (Ed.), Source localization: Continuing discussion on the inverse problem. ISBET Newsletter, 6, 16-28.

Pascual-Marqui, R. D. (1999). Review of methods for solving the EEG inverse problem. International Journal of Bioelectromagnetism, 1 (1), 75-86.

Pascual-Marqui, R. D. (2002). Standardized low resolution brain electromagnetic tomography (sLORETA): Technical details. Methods and Findings in Experimental \& Clinical Pharmacology, 24D, 5-12.

Pascual-Marqui, R. D., Lehmann, D., Koenig, T., Kochi, K., Merlo, M. C. G., Hell, D., et al. (1999). Low-resolution brain electromagnetic tomography (LORETA) functional imaging in acute, neuroleptic-naïve, first-break, productive schizophrenics. Psychiatry Research in Neuroimaging, 90, 169-179.

Pascual-Marqui, R. D., Esslen, M., Kochi, K., \& Lehmann, D. (2002a). Functional imaging with low-resolution brain electromagnetic tomography (LORETA): A review. Methods \& Findings in Experimental \& Clinical Pharmacology, 24C, 91-95.

Pascual-Marqui, R. D., Esslen, M., Kochi, K., \& Lehmann, D. (2002b) Functional imaging with low-resolution brain electromagnetic tomography (LORETA): Review, new comparisons, and new validation. Japanese Journal of Clinical Neurophysiology, 30, 81-94.

Pascual-Marqui, R. D., Michel, C. M., \& Lehmann, D. (1994). Low resolution electromagnetic tomography: A new method for localizing electrical activity in the brain. International Journal of Psychophysiology, 18, 49-65.

Pesarin, F. (2001). Multivariate permutation tests. New York: John Wiley \& Sons.

Pizzagalli, D., Hendrick, A. M., Horras, K. A., \& Davidson, R. R. (2002). Anterior cingulate theta activity is associated with degree of treatment in major depression. International Congress Series, 1232, 711-717.

Pizzagalli, D., Oakes, T. R., \& Davidson, R. J. (2003). Coupling of theta and glucose metabolism in the human rostral anterior cingulate cortex: An EEG/PET study of normal and depressed subjects. Psychophysiology, 40, 1-11.

Prichep, L. S., John, E. R., \& Tom, M. (2001). Localization of deep white matter lymphoma using VARETA: A case study. Clinical Electroencephalography, 32, 62-66.

Rosenzweig, M, Leiman, A., \& Breedlove, S. M. (1996). Biological psychology (pp. 385-390, 584-620). Sunderland, MA: Sinauer Associates, Inc. 
Savander, V., LeDoux, J. E., \& Pitkänen, A. (1996). Topographic projections from the periamygdaloid cortex to select sub regions of the lateral nucleus of the amygdala in the rat. UKU Neuroscience Publications, Neuroscience Letter, 211, 167-170.

Talairach, J., \& Tournoux, P. (1988). Co-planar stereoaxic atlas of the human brain. New York: Theme Medical Publishers.

Thornton, K. E. (1996). On the nature of artifacting the qEEG. Journal of Neurotherapy, 1 (3), 31-39.

Towle, V. L., Bolaños, J., Suarez, D., Tan, K., Grzeszczuk, R., Levin, D. N., et al. (1993). The spatial location of EEG electrodes: Locating the best fitting sphere relative to cortical anatomy. Electroencephalography and Clinical Neurophysiology, $86,1-6$.

Wager, T. D. K., Phan, L., Liberzon, I., \& Taylor, S. F. (2003). Valence, gender and lateralization of functional brain anatomy in emotion: A meta-analysis of findings from neuroimaging. Neuroimage, 19, 513-531.

Westfall, P. H., \& Young S. S. (1993). Resampling-based multiple testing. Examples and methods for p-values adjustment. New York: John Wiley \& Sons.

Worrell, G. A., Lagerlund, T. D., Sharbrough F. W., Brinckmann, B. H., Bucacker, N. E., Cicora, K. M., et al. (2000). Localization of the epileptic focus by low-resolution electromagnetic tomography in patients with a lesion demonstrated by MRI. Brain Topography, 12, 273-282.

RECEIVED: $12 / 17 / 03$

REVISED: 02/23/04

$06 / 14 / 04$

ACCEPTED: 07/07/04 